\title{
ESTRÉS Y DEPRESIÓN EN EL ENTORNO ACADÉMICO-SOCIAL DURANTE EL 2020 EN ESTUDIANTES DE ODONTOLOGÍA
}

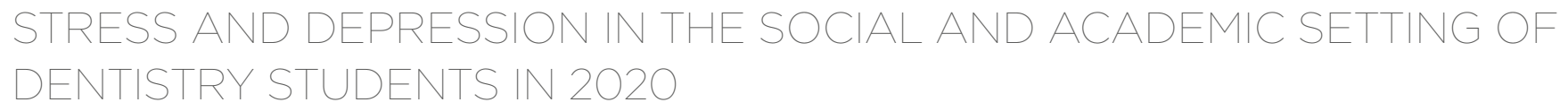

Francis Valeria Espinoza Vilela' * (i)

francisespinoza030798@gmail.com

Carol Magaly Cárdenas Flores ${ }^{1}$ (i) carytocf@gmail.com

Artículo recibido: 13/08/2021

Arbitrado por pares

Artículo aceptado: 24/11/2021

Artículo publicado: 15/12/2021

* Autor corresponsal:

Francis Valeria Espinoza Vilela francisespinoza030798@gmail.com

(cLas autoras, 2021. Publicado por la Universidad Científica del Sur (Lima, Perú)

\section{RESUMEN}

Objetivo: La nueva modalidad de clases virtuales que reciben los estudiantes de odontología puede traer efectos negativos para la salud psicológica o el desempeño académico. El objetivo fue determinar la relación del estrés y depresión en el entorno académico social durante el 2020 en los estudiantes de Odontología de la Universidad Científica del Sur. Materiales y métodos: El estudio fue de tipo descriptivo observacional, transversal y prospectivo, en el que participaron 108 estudiantes de la carrera de Odontología de $6 .^{\circ}$ a $10 .^{\circ}$ ciclo de la Universidad Científica del Sur. Los participantes fueron evaluados mediante un cuestionario con la escala SISCO para medir el estrés académico y la escala BECK (IDB), que se utilizó para evaluar la depresión. Resultados: En el $6 .^{\circ}$ ciclo existe probabilidad de estrés y depresión; el grupo etario más afectado fueron los estudiantes de 19 a 22 años. El valor p para la relación entre el estrés académico y depresión fue $\mathrm{p}$ < 0,001 Conclusión: Existen diferencias estadísticamente significativas entre el estrés académico y la depresión en los estudiantes del $6 .^{\circ}$ al $10{ }^{\circ}$ ciclo de la carrera de Estomatología de la Universidad Científica del Sur.

Palabras claves: estrés, depresión, COVID-19, estudiantes, odontología

\section{ABSTRACT}

Aim: The new modality of virtual classes undertaken by dental students can have negative effects on psychological health and / or academic performance. The objective of this study was to determine the relationship between stress and depression in the social academic environment in dentistry students at the Científica del Sur University in 2020. Materials and Methods: The study was descriptive, observational, cross-sectional, and prospective. 108 students from 6th to 10th cycle of Dentistry at the Universidad Cientifica del Sur participated and were evaluated by means of a questionnaire with the SISCO Inventory to measure academic stress. The BECK scale (BID II) was also used for the evaluation of depression. Results: Students in the 6th term presented a greater probability of stress and depression. The age group most affected was students between 19 and 22 years of age. The $\mathrm{p}$ value for the relationship between academic stress and depression was $\mathrm{p}<0.001$ Conclusion: There are statistically significant differences between academic stress and depression in students from the 6th to the 10th term of the Stomatology career at the Universidad Cientifica del Sur.

Keywords: stress, depression, COVID-19, students, dentistry

Citar como: Espinoza F y Cárdenas C. Estrés y depresión en el entorno académicosocial durante el 2020 en estudiantes de Odontología. Rev Cient Odontol (Lima). 2021; 9(4): e080.

DOI: 10.21142/2523-2754-0904-2021-080

1 Carrera de Estomatología, Universidad Científica del Sur. Lima, Perú. 


\section{INTRODUCCIÓN}

Debido a la COVID-19, la población peruana viene atravesando cuadros de estrés y depresión por el nuevo estilo de vida y restricciones que plantea el Estado. Uno de los cambios más radicales ha sido implementar la modalidad de enseñanza virtual en colegios, institutos y universidades $\left({ }^{1}\right)$. La carrera de Odontología se caracteriza por ser exigente y de ámbito teórico-práctico, ya que en los últimos años de carrera se realizan tratamientos en pacientes. Bajo la nueva modalidad, los estudiantes de dicha carrera vienen afrontando clases 100\% teóricas, pese a la necesidad de tener horas prácticas. Este factor, así como la sobrecarga de trabajos, tareas, exámenes y clase de horas prolongadas, pueden afectar la salud psicológica o el desempeño en sus clases $\left({ }^{2-4}\right)$.

Es probable que los trabajos, exámenes y horas prolongadas de clases virtuales estén preocupando y demandando mucho tiempo semanal de los estudiantes, lo que influye en su estado anímico y desenvolvimiento en las clases ya que, a pesar de estar en casa todos los días, invierten más de 40 horas en promedio de clases teóricas semanales, las cuales pueden abarcar de 8-10 horas diarias sin tomar en cuenta el tiempo que utilizan realizando trabajos y tareas fuera del horario curricular ${ }^{(5-7)}$. Esta carga académica se viene manifestando en los estudiantes con varios síntomas que conllevan al estrés y la depresión, ya que bajo el aislamiento social no les permite despejarse ni los domingos, a pesar de estar seis días de la semana dedicados la mayor parte del tiempo a los estudios $\left({ }^{8-10}\right)$.

Ante esta situación, es muy importante tener estrategias que permitan reducir los niveles de estrés y depresión que aparecen en la vida de los universitarios. El objetivo del estudio fue determinar la relación del estrés y la depresión con el entorno académico-social durante el 2020 en los estudiantes de Odontología de la Universidad Científica del Sur.

\section{MATERIALES Y MÉTODOS}

El estudio fue de tipo descriptivo-observacional, prospectivo y transversal. La población de estudio comprendió a los estudiantes de la carrera de Estomatología de la Universidad Científica del Sur. La muestra se realizó mediante la fórmula de proporción Fisterra. Se incluyó a los estudiantes de manera aleatoria, sin antecedentes de enfermedades psicológicas, que cursaban del $6 .^{\circ}$ al $100^{\circ}$ ciclo de la carrera durante el 2020, y que decidieron participar en el estudio y firmaron el consentimiento informado. Se les aplicó dos instrumento de evaluación mediante el uso de dos cuestionarios validados en el país, escala SISCO y escala de Beck.

\section{Instrumento de Evaluación Estrés SISCO}

E1 cuestionario SISCO consta de 31 preguntas objetivas que permiten determinar en qué categoría se encontraron los participantes, ya sea bajo (0-79), medio (80-88), medio (88) o alto (89-180), mediante la sumatoria e interpretación según la respuesta del participante, teniendo en cuenta una escala del 1 al 5, en la que 1 es Nunca, 2 es Rara vez, 3 es Algunas veces, 4 es Casi siempre y 5 es Siempre.

\section{Instrumento de Evaluación Depresión Beck (IDB)}

El cuestionario IDB consta de 21 preguntas objetivas que permiten determinar en qué categoría se encuentran los participantes: Altibajos considerados normales (010), Leve perturbación del estado de ánimo (11-16), Estados de depresión intermitentes (17-20), Depresión moderada (21-30) o Depresión grave (31-40), una vez completado el cuestionario.

Se sumaron los puntos correspondientes a cada una de las 21 preguntas y se obtuvo el total con la finalidad de medir tanto el estrés académico como la depresión en los alumnos que cursaban del $6 .^{\circ}$ al $10 .^{\circ}$ ciclo de la carrera. El investigador se calibró en el empleo de los cuestionarios indicados y se realizó la prueba piloto al $10 \%$ de la muestra que indican los antecedentes con un Kappa = 0,85 para hallar el tamaño de muestra se utilizó el estimador de tamaño muestral de proporción Fisterra $\mathrm{N}=108$. 
La investigación fue evaluada por el Comité de Ética de la Universidad Científica del Sur, los correos electrónicos proporcionados serán confidenciales y no serán utilizados para otros fines que no sean parte de la investigación, a fin de proteger la confidencialidad del participante.

Tabla 1. Relación del grado de estrés (SISCO) según edad

\begin{tabular}{lccccc}
\multicolumn{1}{c}{ Escala } & \multicolumn{3}{c}{ Grupo etario } & Total & Valor p \\
Sisco & $\mathbf{1 9 - 2 2}$ & $\mathbf{2 3 - 2 5}$ & Más de 26 & & \\
Bajo & $20(18,5 \%)$ & $16(14,8 \%)$ & $5(4,6 \%)$ & $41(38 \%)$ & \\
Medio & $10(9,3 \%)$ & $8(7,4 \%)$ & $8(7,4 \%)$ & $26(24,1 \%)$ & $0,436 *$ \\
Alto & $21(19,4 \%)$ & $13(12 \%)$ & $7(6,5 \%)$ & $41(38,0 \%)$ & \\
\hline Total & $51(47,2 \%)$ & $37(34,3 \%)$ & $20(18,5 \%)$ & $108(100 \%)$ & \\
\hline
\end{tabular}

*Prueba exacta de Fisher

Tabla 2. Relación del grado de depresión (Beck) según edad

\begin{tabular}{lccccc}
\multicolumn{1}{c}{$\begin{array}{c}\text { Escala } \\
\text { Beck }\end{array}$} & $\mathbf{1 9 - 2 2}$ & $\mathbf{2 3 - 2 5}$ & Más de 26 & Total & Valor p \\
Normal & $25(23,1 \%)$ & $24(22,2 \%)$ & $12(11,1 \%)$ & $61(56,5 \%)$ & \\
Leve & $12(11,1 \%)$ & $7(6,5 \%)$ & $4(3,7 \%)$ & $23(21,3 \%)$ & $0,715 *$ \\
Moderado & $12(11,1 \%)$ & $6(5,6 \%)$ & $3(2,8 \%)$ & $21(19,4 \%)$ & \\
Extremo & $2(1,9 \%)$ & $0(0 \%)$ & $1(0,9 \%)$ & $3(2,8 \%)$ & \\
Total & $51(47,2 \%)$ & $37(34,3 \%)$ & $20(18,5 \%)$ & $108(100 \%)$ & \\
\hline
\end{tabular}

*Prueba exacto de Fisher

Tabla 3. Relación del grado de estrés (SISCO) según ciclo

\section{RESULTADOS}

El plan estadístico se realizó utilizando el sistema SPSS (Inc Armonk NY, EE. UU.) para evaluar las variables estrés académico y depresión en estudiantes de odontología de $6 .^{\circ}$ a $10 .^{\circ}$ ciclo de la Universidad Científica del Sur. La muestra estuvo compuesta por 108 alumnos, divididos en 3 grupos etarios: 19-22 años, 2325 años y 26 años a más.

Se evaluó la relación de estrés (escala SISCO) y el grupo etario, y se encontró estrés alto en el grupo de 19 a 22 años (19,4\%); no mostró diferencias estadísticamente significativas, con un $\mathrm{p}=0,436$ (tabla 1 ). Al evaluar la depresión, se encontró depresión moderada en el grupo etario de 19 a 22 años (11,1\%), y ausencia de depresión en el $56,5 \%$ del total de la muestra. No se hallaron diferencias estadísticamente significativas $p=0,715$ (tabla 2).

Al evaluar la relación de estrés (escala SISCO) y el ciclo de estudios, se encontró estrés alto $(10,2 \%)$ en los estudiantes que cursan el VII ciclo; no mostró diferencias estadísticamente significativas, con un $\mathrm{p}=$ 0,194 (tabla 3). $\mathrm{Al}$ evaluar la depresión, se encontró depresión leve en el $5,6 \%$ de los estudiantes que cursan el VI ciclo, y ausencia de depresión en el 56,5\%. No se encontraron diferencias estadísticamente significativas $\mathrm{p}=0,679$ (tabla 4).

\begin{tabular}{|c|c|c|c|c|c|c|c|}
\hline \multirow{2}{*}{ Escala SISCO } & \multicolumn{5}{|c|}{ Ciclo de estudio } & \multirow{2}{*}{ Total } & \multirow{2}{*}{ Valor $\mathbf{p}$} \\
\hline & Ciclo VI & Ciclo VII & Ciclo VIII & Ciclo IX & Ciclo X & & \\
\hline Bajo & $16(14,8 \%)$ & $8(7,4 \%)$ & $4(3,7 \%)$ & $6(5,6 \%)$ & $7(6,5 \%)$ & $4 I(38 \%)$ & \multirow{3}{*}{$0,194 *$} \\
\hline Medio & $3(2,8 \%)$ & $6(5,6 \%)$ & $4(3,7 \%)$ & $5(4,6 \%)$ & $8(7,4 \%)$ & $26(24 \%)$ & \\
\hline Alto & $7(6,5 \%)$ & $8(7,4 \%)$ & II (I0,2\%) & $7(6,5 \%)$ & $8(7,4 \%)$ & $4 I(48 \%)$ & \\
\hline Total & $26(24,1 \%)$ & $22(20,4 \%)$ & $19(17,5 \%)$ & | $8(16,7 \%)$ & $23(21,3 \%)$ & $108(100 \%)$ & \\
\hline
\end{tabular}

*Prueba exacto de Fisher

Tabla 4. Relación del grado de depresión (Beck) según ciclo

\begin{tabular}{|c|c|c|c|c|c|c|c|}
\hline \multirow{2}{*}{ Escala Beck } & \multicolumn{5}{|c|}{ Ciclo de estudio } & \multirow{2}{*}{ Total } & \multirow{2}{*}{ Valor $\mathrm{p}$} \\
\hline & Ciclo VI & Ciclo VII & Ciclo VIII & Ciclo IX & Ciclo X & & \\
\hline Normal & $15(13,8 \%)$ & $15(13,8 \%)$ & $8(7,4 \%)$ & $9(8,3 \%)$ & $14(13,0 \%)$ & $6 \mathrm{I}(56,5 \%)$ & \multirow{3}{*}{$0,679 *$} \\
\hline Moderado & $5(4,6 \%)$ & I (0,9\%) & $7(6,5 \%)$ & $3(2,8 \%)$ & $5(4,6 \%)$ & $21(19,4 \%)$ & \\
\hline Extremo & $0(0 \%)$ & I $(0,9 \%)$ & I $(0,9 \%)$ & I $(0,9 \%)$ & $0(0 \%)$ & $3(2,8 \%)$ & \\
\hline
\end{tabular}

*Prueba exacto de Fisher 
Tabla 5. Relación entre estrés y depresión en estudiantes de la carrera de Odontología

\begin{tabular}{|c|c|c|c|c|c|}
\hline \multirow{2}{*}{$\begin{array}{c}\text { Escala } \\
\text { Beck }\end{array}$} & \multicolumn{3}{|c|}{ Escala SISCO } & \multirow{2}{*}{ Total } & \multirow{2}{*}{$\mathbf{p}$} \\
\hline & Bajo & Medio & Alto & & \\
\hline Normal & $34(31,5 \%)$ & $17(15,7 \%)$ & $10(9,2 \%)$ & $6 \mathrm{I}(56,4 \%)$ & \multirow{5}{*}{$<0,00$ । } \\
\hline Leve & 7 (6,5\%) & $8(7,4 \%)$ & $8(7,4 \%)$ & $23(21,3 \%)$ & \\
\hline Moderado & $0(0 \%)$ & I $(0,9 \%)$ & $20(\mid 8,5 \%)$ & $21(19,4 \%)$ & \\
\hline Extrema & $0(0 \%)$ & $0(0 \%)$ & $3(2,8 \%)$ & $3(2,8 \%)$ & \\
\hline Total & 41 (38\%) & 26 (24\%) & $4 \mid(37,9 \%)$ & $108(100 \%)$ & \\
\hline
\end{tabular}

*Prueba exacta de Fisher

Al evaluar el estrés y la depresión en los estudiantes del VI al X ciclo de la Carrera de Odontología de la Universidad Científica del Sur, se halló que la depresión moderada con estrés alto representó un 18,5\% del total, y sin depresión y estrés bajo, un $31,5 \%$, con diferencias estadísticamente significativas: $\mathrm{p}<0,001$ (tabla 5).

\section{DISCUSIÓN}

El estrés y la depresión en estudiantes de ciencias de la salud y otras carreras universitarias se viene percibiendo desde hace varias décadas, y suelen estar asociados con factores académicos, psicosociales, laborales y económicos $\left({ }^{11,12}\right)$. Algunos estudios señalan que, en la carrera de Odontología, el porcentaje de estrés académico varía entre un 38 y un 40\%, y se relaciona con la alta carga académica, los excesivos trabajos o tareas para la casa, los exámenes, el miedo a reprobar y las prolongadas horas de estudio semanal $\left({ }^{12}\right)$. Hoy en día, el estrés se ha convertido en un problema a nivel mundial que genera grandes problemas psicológicos e incrementa la tasa de mortalidad incluso en países desarrollados $\left({ }^{13}, 14\right)$. Esto propicia el inicio de enfermedades sistémicas en los estudiantes. Por ello, resulta importante prestar atención a los síntomas y los cambios emocionales que puede presentar el estudiante universitario, ya que esto puede afectar su desempeño académico, sobre todo ante la crisis sanitaria que venimos atravesando por la COVID-19 $(2,15)$. El cambio en la modalidad de estudios que los estudiantes de odontología experimentan les causa una gran frustración $\left({ }^{16}\right)$.

En el 2018, se realizó un estudio sobre una universidad privada de Lima con 140 participantes, quienes fueron evaluados mediante el cuestionario Dental Environment Stress Questionaire, tomando en consideración el estrés, la edad, el sexo y el ciclo que cursaban. Se obtuvo como resultado que el 75,7\% de los participantes padecía de estrés académico, con una relación significativa entre el ciclo y la edad del participante que repercutía más en los ciclos preclínicos y en los jóvenes más que en los adultos $\left.{ }^{17}\right)$. Al comparar esos resultados con los del presente estudio, se halló que todos los participantes tenían estrés académico, pero en este caso no se encontró una relación significativa entre la edad y ciclo. Podemos afirmar que el estrés académico se presentó más en la población de 19 a 22 años y que el estrés es más frecuente en los estudiantes más jóvenes. En relación con el estrés académico y el ciclo no se pudo encontrar una relación ya que solo se tomó la muestra del $6 .^{\circ}$ al $10 .^{\circ}$ ciclo, que corresponde a cursos clínicos; pese a ello, se evidenció una mayor carga de estrés en el $7 .^{\circ}$ ciclo.

En el ámbito científico internacional, en el 2016, se realizó un estudio en estudiantes de medicina de la Universidad de Fayoum, en el cual se evaluó a los estudiantes mediante la escala Depression, Anxiety and Stress Scale-21 (DASS-21). La prevalencia de estrés fue del 62,4\%; la de ansiedad, del 64,3\%; y la de depresión, del 60,8\% en los participantes. Los puntajes más altos de estrés y ansiedad estaban asociados con el sexo femenino y la edad avanzada $\left({ }^{18}\right)$. En el caso del presente estudio, fue realizado en estudiantes de Odontología y estos fueron evaluados mediante la escala de SISCO para estrés académico y la escala de Beck para depresión. El nivel de estrés fue mayor en dicha población, ya que todos los estudiantes lo padecían, ya sea en un nivel bajo, medio o alto; sin embargo, el 100\% de la muestra presentaba depresión y el 47,2\% de esta afección se presentó en estudiantes 19 a 22 años.

En el 2020, se realizó un estudio transversal en Arabia Saudita con la finalidad de evaluar el impacto psicológico de los estudiantes de Odontología utilizando el cuestionario Depression, Anxiety and stress scale-21, mediante el cual se encontró que el $60,6 \%$ de los estudiantes tenía depresión; el 37\%, ansiedad; y 34,9\%, estrés $\left({ }^{19}\right)$. En el presente estudio, el 46,5\% tuvo depresión y el 37,9\%, estrés académico alto. Si estos resultados los comparamos con el estudio realizado en el $2016\left({ }^{18}\right)$, 
podemos evidenciar que la tasa de depresión entre los estudiantes de Odontología y Medicina no ha tenido diferencias significativas; sin embargo, si lo comparamos con el estudio en una universidad peruana del 2018, podemos evidenciar que el porcentaje de estrés en los estudiantes fue del $75,7 \%$.

El presente estudio tuvo como principal limitante la cantidad de alumnos participantes; al no alcanzar la muestra ideal, se procedió a incluir en el estudio a estudiantes del VI cuatrimestre. La muestra se tuvo que dividir en intervalos por conveniencia en la sección edad para que no varíe el porcentaje de afectados y no existan sesgos en la medición y los resultados. Este estudio tiene una gran importancia académica para cada estudiante y docente de la carrera; ya que al saber que existe una tasa considerable de estrés y depresión se pueden aplicar estrategias para contrarrestar su incidencia a través de clases más didácticas, un servicio de psicología y orientación para que los estudiantes sepan cómo controlar el estrés y la incertidumbre por la excesiva carga académica y la nueva modalidad de estudio que afronta la educación en el Perú, al ser 100\% virtual. El apoyo profesional adecuado permitirá adecuar las estrategias específicas para minimizar o incluso erradicar el estrés propiciando el equilibrio y organización de las obligaciones.

\section{CONCLUSIONES}

Existen relación entre el estrés académico y la depresión en los estudiantes del $6 .^{\circ}$ al $10 .^{\circ}$ ciclo de la carrera de Odontología de la Universidad Científica del Sur. No se encontró relación entre el nivel de depresión y de estrés con la edad y el ciclo de estudio de los estudiantes.

Contribución de los autores: Francis Valeria Espinoza Vilela realizó el diseño y la ejecución el estudio, aportes en la recolección de datos, redactó el borrador inicial y la versión final.

Carol Cárdenas Flores concibió la idea y el diseño del estudio, realizó la interpretación de datos, revisó el borrador inicial y revisó y aprobó la versión final.

Fuente de financiamiento: Autofinanciado.

Conflicto de intereses: Los autores declaran no tener conflicto de intereses. 


\section{REFERENCIAS BIBLIOGRÁFICAS}

1. Sabino-Silva R, Jardim ACG, Siqueira WL. Coronavirus COVID-19 impacts to dentistry and potential salivary diagnosis. Clin Oral Investig. 2020; 24(4): 1619-21. doi:10.1007/s00784020-03248

2. Misrachi-Launert C, Ríos-Erazo M, Manríquez-Urbina JM, Burgos-Ibarra C, Ponce-Espinoza D. Fuentes de estrés percibidas y rendimiento académico de estudiantes de odontología chilenos. FEM 2015; 18(2). doi:10.4321/S2014-98322015000200006

3. Evaristo-Chiyong T, Chein-Villacampa S. Estrés y desempeño académico en estudiantes de Odontología. Odontol Sanmarquina. 2015; 18(1): 23. doi:10.15381/os.v18i1.11336

4. Rodríguez de los Ríos LA, Carbajal YM, Narváez T, Gutiérrez RJ. Impacto emocional por COVID-19 en estudiantes universitarios. Un estudio comparativo. EducaUMCH. 2020; (16): 14. https:// doi.org/10.15366/Educa UMCH2019.17.3.001

5. Stormon N, Ford PJ, Kisely S, Bartle E, Eley DS. Depression, anxiety and stress in a cohort of Australian dentistry students. Eur J Dent Educ.2019; 23(4): 507-14. doi:10.1111/eje.12459

6. Oblitas Guadalupe LA, Soto Vásquez DE, Anicama Gómez JC, Arana Sánchez AA. Incidencia del mindfulness en el estrés académico en estudiantes universitarios: Un estudio controlado. Ter Psicológica. 2019; 37(2): 116-28. doi:10.4067/S071848082019000200116

7. González-Olaya HL, Delgado-Rico HD, Escobar-Sánchez M, Cárdenas-Angelone ME. Asociación entre el estrés, el riesgo de depresión y el rendimiento académico en estudiantes de los primeros semestres de un programa colombiano de medicina. FEM 2014; 17(1): 47-54. https://dx.doi.org/10.4321/S201498322014000100008

8. Bresolin JZ, Dalmolin G de L, Vasconcellos SJL, Barlem ELD, Andolhe R, Magnago TSB de S. Depressive symptoms among healthcare undergraduate students. Rev Latino-Am Enfermagem. 2020; 28: e3239. doi: 10.1590/1518-8345.3210.3239

9. González L. Estrés académico en estudiantes universitarios asociados a la pandemia por COVID-19. I+D. 2020; 9(25): 15879. https://doi. org/10.31644/IMASD.25.2020.a10
10. Apaza CM, Seminario RS, Santa-Cruz JE. Factores psicosociales durante el confinamiento por el Covid-19 - Perú. Rev Venez Gerenc. 2020; 25(90): 402-13. https://doi.org/10.37960/rvg. v25i90.32385

11. Vizoso Gómez CM, Arias Gundín O. Estresores académicos percibidos por estudiantes universitarios y su relación con el burnout y el rendimiento académicos. Anu Psicol. 2016; 46(2): 90-7. doi:10.1016/j.anpsic.2016.07.006

12. Castillo Pimienta C, Chacón de la Cruz T, Díaz-Véliz G. Ansiedad y fuentes de estrés académico en estudiantes de carreras de la salud. Investigación en Educación Médica 2016; 5(20): 230-7. http://dx.doi.org/10.1016/j.riem.2016.03.001

13. Perea LAH, Téllez JES, González GIM, Esmeralda O, Luis R. Percepción de estrés en universitarios, su impacto en el desempeño académico, afrontamiento y apoyo familiar. 2016; 3(2): 10 .

14. Ramachandiran M, Dhanapal S. Academic stress among university students: a quantitative study of Generation $\mathrm{Y}$ and Z's perception. Pertanika J Soc Sci Hum. 2018; 26(3): 2115-28.

15. Hj Ramli N, Alavi M, Mehrinezhad S, Ahmadi A. Academic Stress and Self-Regulation among University Students in Malaysia: Mediator Role of Mindfulness. Behav Sci. 2018; 8(1): 12. doi:10.3390/bs8010012

16. Vergara-Buenaventura A, Chavez-Tuñon M, Castro-Ruiz C. The Mental Health Consequences of COVID-19 pandemic in Dentistry. Disaster Med Public Health Prep. 2020; 1-13. doi:10.1017/dmp.2020.190

17. Córdova Sotomayor DA, Santa Maria Carlos FB. Factores asociados al estrés en estudiantes de odontología de una universidad peruana. Rev Estomatol Herediana. 2018; 28(4): 252. https://doi.org/10.20453/reh.v28i4.3429

18. Abdel Wahed WY, Hassan SK. Prevalence and associated factors of stress, anxiety and depression among medical Fayoum University students. Alexandria Journal of Medicine. 2017; 53(1): 77-84. doi: 10.1016/j.ajme.2016.01.005

19. Hakami Z, Khanagar SB, Vishwanathaiah S, et al. Psychological impact of the COVID-19 pandemic on dental students: A Nationwide Study. J Dent Educ. 2020; 1-10. https://doi. org/10.1002/jdd. 12470 